\title{
Analysis of Stresses and Deflection of Sun Gear by Theoretical and ANSYS Method
}

\author{
Yogesh C. Hamand, Vilas Kalamkar \\ Mechanical Department, Sardar Patel College of Engineering, Mumbai, India \\ E-mail:yogeshhamand@rediffmail.com,vilaskalamkar@gmail.com \\ Received September 30, 2011; revised October 25, 2011; accepted November 5, 2011
}

\begin{abstract}
Gearing is one of the most critical components in mechanical power transmission systems. This article examines the various stresses and deflection developed in sun gear tooth of planetary gearbox which is used in Grabbing Crane. Article includes checking sun gear wear stresses and bending stresses using IS 4460 equations. Also calculate various forces acting on gear tooth. In this study, perform the calculation for sun gear tooth to calculate bending, shear, wear \& deflection using theoretical method. 3D model is created of circular root fillet \& trochoidal root fillet of gear tooth for simulation using ProE Wildfire 3. In Pro-E, the geometry is saved as a file and then it is transferred from Pro-E to ANSYS 10 in IGES format. The results of the $3 \mathrm{D}$ analyses from ANSYS are compared with the theoretical values. Comparison of ANSYS results in circular root fillet $\&$ trochoidal root fillet also carry out.
\end{abstract}

Keywords: Bending Stress, Circular Root Fillet, Deflection, Grabbing Crane, Planetary Gearbox, Shear Stress, Sun Gear, Trochoidal Root Fillet, Wear

\section{Introduction}

In spite of the number of investigations devoted to gear research and analysis there still remains to be developed, a general numerical approach capable of predicting the effects of variations in gear geometry, shear, wear and bending stresses. The objective of this work is to use ANSYS to develop theoretical models of the behavior of planetary gears in mesh, to help to predict the effect of gear tooth stresses and deflection. The main focus of the current research as developed here is to develop and to determine appropriate models of contact elements, to calculate various stresses and using ANSYS and compare the results with theoretical.

The project work mainly deals with

1) Checking of wear stresses \& bending stresses using IS 4460 equations for sun gear.

2) Force calculations for planetary gear

3) Calculate the values for sun gear tooth for bending, shear, wear \& deflection using theoretical method.

4) Generation of gear tooth profile in Pro-E3.

5) Create 3D model of circular root fillet \& trochoidal root fillet of gear tooth for simulation using Pro-E3.

6) Importing Pro-E model in ANSYS in IGES format.

7) Comparison of the results of the $3 \mathrm{D}$ analyses from ANSYS with the theoretical values.

8) Comparison of ANSYS results in circular root fillet
\& trochoidal root fillet.

Shanmugasundaram Sankar, Maasanamuthu Sundar Raj \& Muthusamy Nataraj [1] have introduced Corrective measures are taken to avoid tooth damage by introducing profile modification in root fillet. Tesfahunegn and Rosa [2] investigated the influence of the shape of profile modifications on transmission error, root stress and contact pressure through non linear finite element approach. Chun-Fang Tsai ,Tsang-Lang Liang \& Shyue-Cheng Yang [3] prepared a complete mathematical model of the planetary gear mechanism with double circular-arc teeth is developed. Ravichandra Patchigolla and Yesh P. Singh [4] developed a program using ANSYS Parametric De- sign Language (APDL) to generate 1,3 or 5 tooth segment finite element models of a large spur gear. Faydor L. Litvin, Alfonso Fuentes, Daniele Vecchiato, and Ignacio Gonzalez-Perez [5] proposed a new types of planetary and planetary face-gear drives \& the new designs are based on regulating backlash between the gears and modifying the tooth surfaces to improve the design.

\section{Terminology}

\subsection{Terminology-Spur Gears}

Refer: Figure 1. 
Diametral pitch $\left(d_{p}\right)$ : The number of teeth per one inch of pitch circle diameter.

Module (m): The length, in mm, of the pitch circle diameter per tooth.

Circular pitch (p): The distance between adjacent teeth measured along the pitch circle diameter

Addendum $\left(h_{\mathrm{a}}\right)$ : The height of the tooth above the pitch circle diameter.

Centre distance (a): The distance between the axis of two gears in mesh.

Circular tooth thickness (Ctt): The width of a tooth measured along the are at the pitch circle diameter.

Dedendum $\left(\mathrm{h}_{\mathrm{f}}\right)$ : The depth of the tooth below the pitch circle diameter.

Outside diameter $\left(\mathrm{D}_{\mathrm{o}}\right)$ : The outside diameter of the gear.

Base Circle diameter $\left(D_{b}\right)$ : The diameter on which the involute teeth profile is based.

Pitch circle dia (D): The diameter of the pitch circle.

Pitch point: The point at which the pitch circle diameters of two gears in mesh coincide.

Pitch to back: The distance on a rack between the pitch

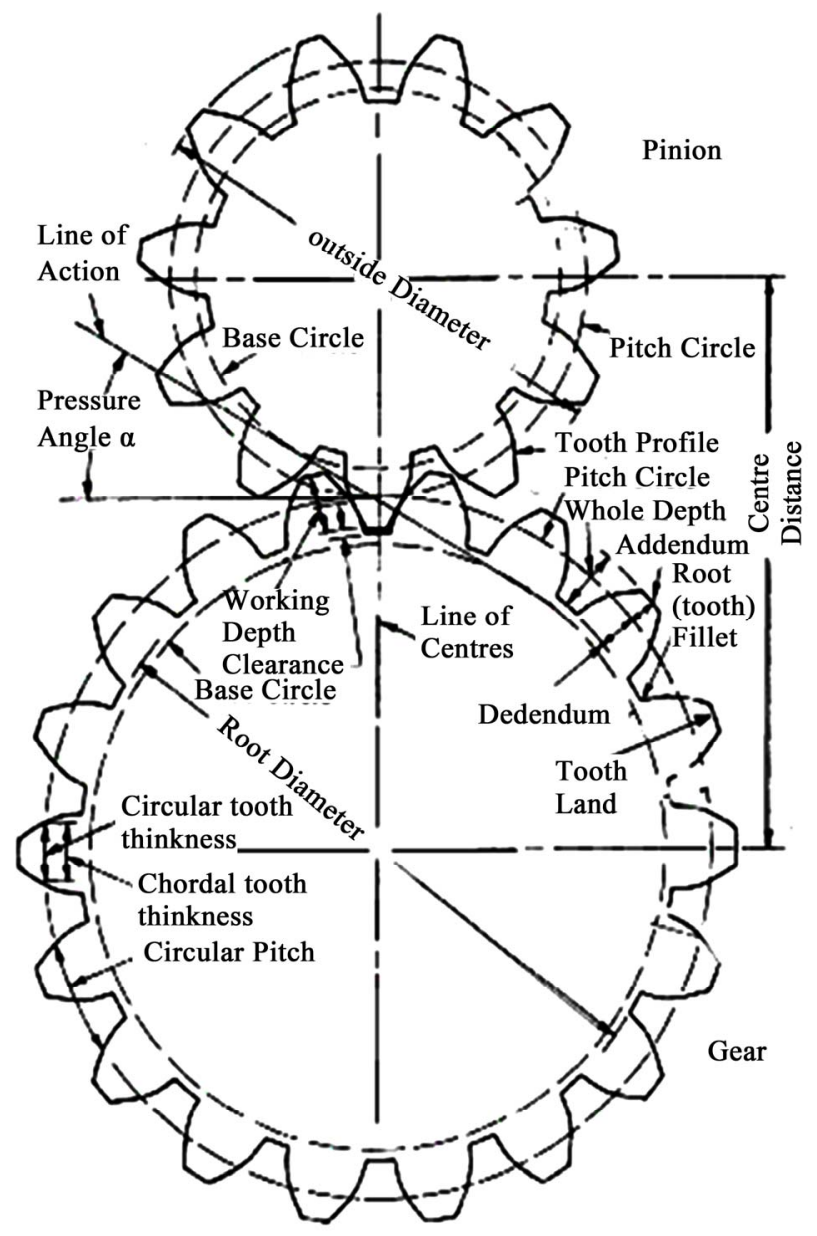

Figure 1. Terminology—spur gears. circle diameter line $\&$ the rear face of the rack.

Pressure angle: The angle between the tooth profile at the pitch circle diameter $\&$ a radial line passing through the same point.

Whole depth: The total depth of the space between adjacent teeth.

\subsection{Terminology—Planetary Gear Train}

\section{Refer Figures 2 and 3.}

Sun: The central gear

Planet Gear: Peripheral gears, of the same size, meshed with the sun gear and Annulus

Planet carrier: Holds one or more peripheral planet gears, of the same size, meshed with the sun gear

Ring Gear/Annulus: An outer ring with inward-facing teeth that mesh with the planet gear or gears

No. of teeth on Gear (Z)

No. of teeth on Sun Gear (S)

No. of teeth on Planet Gear (P)

No. of teeth on Pinion Gear 1 (P1)

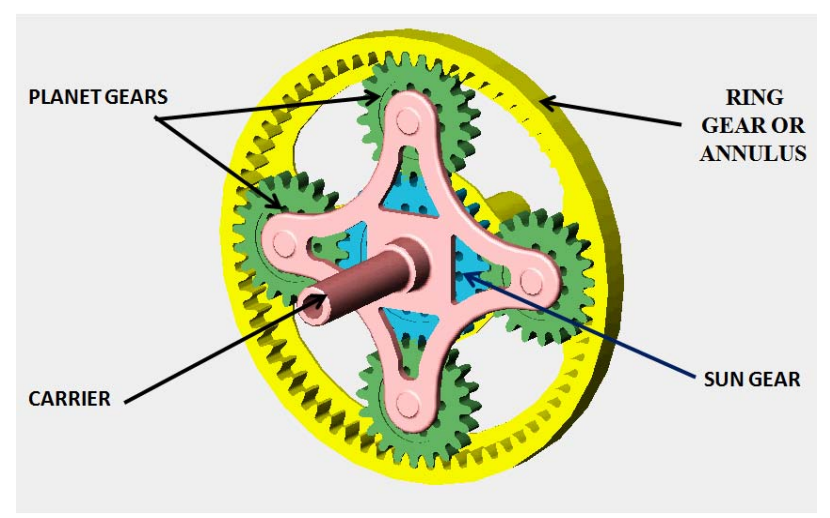

Figure 2. Planetary gear train.

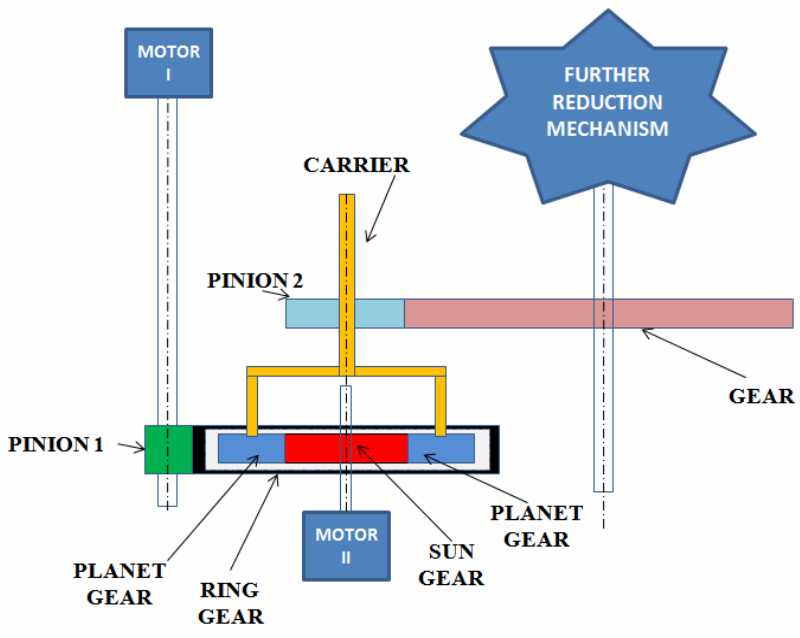

Figure 3. Schematic diagram of planetary gear train. 
No. of teeth on Pinion Gear 2 (P2)

No. of teeth on Ring Gear External (RE)

No. of teeth on Ring Gear Internal (RI)

Total Gear Reduction Ratio (TR)

How Planetary Gear Train works? For different conditions of Motor I \& II in Figure 3, Gear ratios are shown in Table 1.

\section{Grabbing Crane Specification}

For Photograph Refer: Figure 4

Capacity: 10 Ton wire rope (weight of material handled + grab)

Duty: Class 4/12 Hr

Location: Outdoor

Table 1. Gear ratio.

\begin{tabular}{|c|c|c|c|}
\hline $\begin{array}{l}\text { Motor's On \& Off } \\
\text { Condition }\end{array}$ & $\begin{array}{l}\text { Motor "I" On \& } \\
\text { Motor "II" Off }\end{array}$ & $\begin{array}{l}\text { Motor "I" Off \& } \\
\text { Motor "II" On }\end{array}$ & $\begin{array}{l}\text { Motor "I" On \& } \\
\text { Motor "II" On } \\
\text { (Opposite } \\
\text { Direction) }\end{array}$ \\
\hline $1^{\text {st }}$ Stage & $\mathrm{RE} / \mathrm{P} 1$ & ---- & \\
\hline $2^{\text {nd }}$ Stage & $(\mathrm{RI}+\mathrm{S}) / \mathrm{RI}$ & $(\mathrm{RI}+\mathrm{S}) / \mathrm{S}$ & TR \\
\hline $3^{\text {rd }}$ Stage & $\mathrm{G} / \mathrm{P} 2$ & $\mathrm{G} / \mathrm{P} 2$ & $=\frac{\mathrm{TR} 1 \times \mathrm{TR} 2}{\mathrm{TR} 1+\mathrm{TR} 2}$ \\
\hline Total Reduction & $\begin{array}{c}\mathrm{TR} 1=(\mathrm{RE} / \mathrm{P} 1) \times \\
((\mathrm{RI}+\mathrm{S}) / \mathrm{RI}) \times \\
(\mathrm{G} / \mathrm{P} 2)\end{array}$ & $\begin{array}{c}\mathrm{TR} 2=((\mathrm{RI}+ \\
\mathrm{S}) / \mathrm{S}) \times(\mathrm{G} / \mathrm{P} 2)\end{array}$ & \\
\hline
\end{tabular}

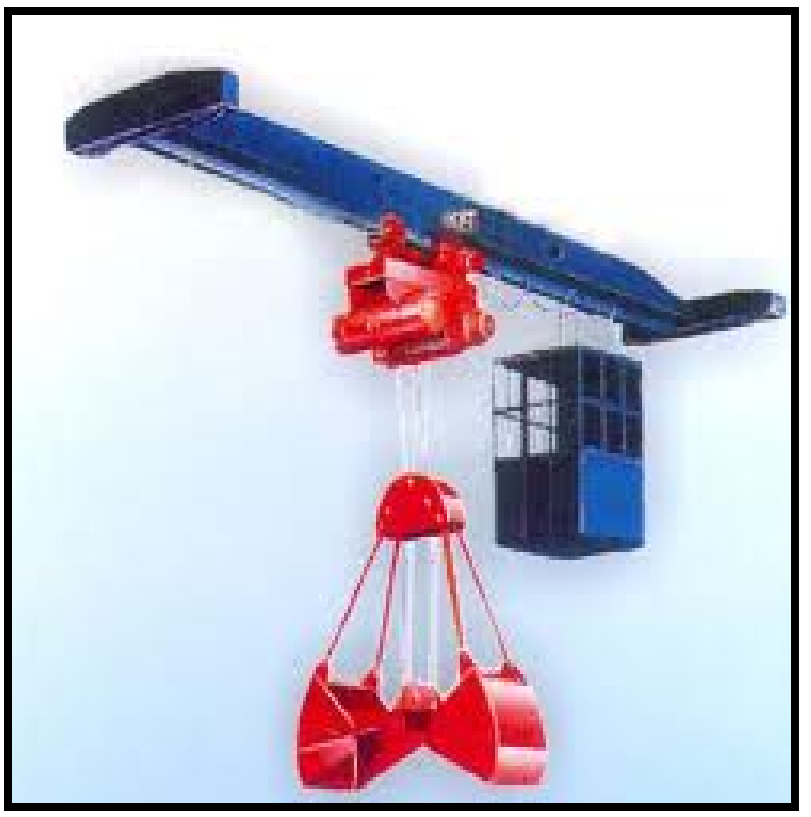

Figure 4. Grabbing crane.
Total weight of trolley: $13 \mathrm{~T}$

Total weight of crane: $71 \mathrm{~T}$

Ambient temperature: $50^{\circ} \mathrm{C}$

Lubrication: Group

Operations from: Closed cabin

Grab bucket capacity: $3.5 \mathrm{~m}^{3}$

Material handled: Blast furnace slag

Bulk density: $1.1-1.2 \mathrm{~T} / \mathrm{m}^{3}$

Weight of material handled: $4 \mathrm{~T}$

Dead weight of grab bucket: $5.5 \mathrm{~T}$

Different Parts in figure 5 mentioned in Table2

\section{Construction of Gearbox}

There are two basic operations involved in this gearbox, which are

1) Holding

2) Opening-closing.

\subsection{Holding}

It is a 3-stage mechanism out of which, first two stages are helical \& third stage which is output of this mechanism is a spur gear pair.

We have used spur gear in output stage because,

1) Due to large P.C.D. of exterior teeth of annulus, it is very difficult to machine helical gearing on it, hence spur teeth are machined.

2) The output shaft of the holding drum is heavily loaded. As per the requirement of high hoist speed, the rotational speeds of the shafts are very high, which leads to selection of large capacity of bearings.

If helical gearing is used in the output of this gearbox, additional thrust factors are generated which further increase the bearing size. Hence, spur is preferred in the output stage of the gearboxes of such applications.

\subsection{Opening-Closing}

It is also a 3-stage mechanism in which, first stage is spur $\&$ rest two are helical. Helical gears are used to achieve desired reduction \& maximum efficiency. Output of this mechanism is given to SUN of "Planetary Gear System"

\subsection{Planetary System}

Planetary system consists of one SUN gear, three PLANETS \& an ANNULUS. When holding motor is put on, SUN is kept fixed \& when opening-closing motor is in operation; annulus is kept fixed.

\section{Working of Gear Box}

The grab bucket is closed as follows (Figure 6(b)). The 


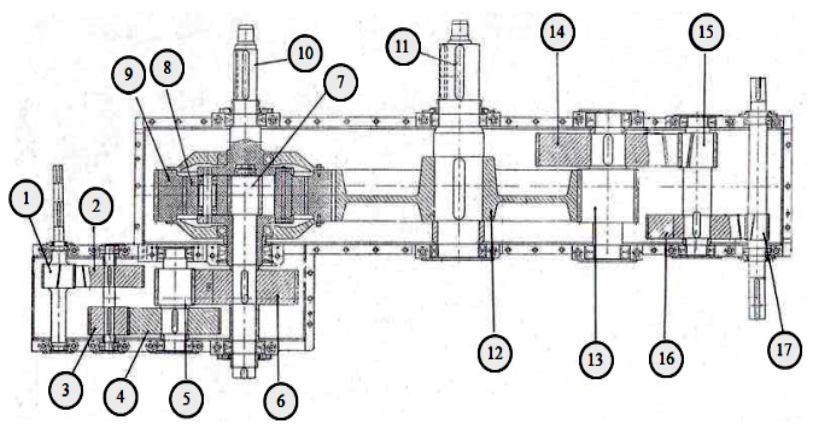

Figure 5. Planetary gears system used in grab crane's hoist.

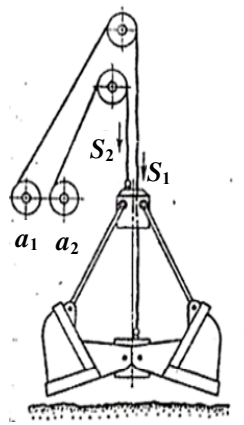

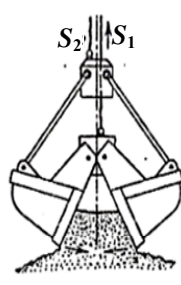

(b)

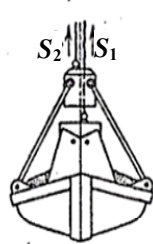

(c)

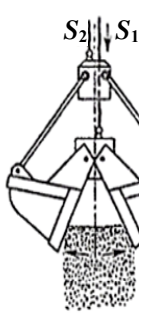

(d)
Figure 6. Principal of operation of a double rope grab bucket.

Table 2. Part list (Refer Figure 5).

\begin{tabular}{|c|c|c|}
\hline $\begin{array}{l}\text { Part } \\
\text { No. }\end{array}$ & Description & Material \\
\hline 1 & Input Helical Pinion $\mathrm{Z}=31 \mathrm{~m}=4(\mathrm{RH})$ & EN24 \\
\hline 2 & Helical Gear Z $=68 \mathrm{~m}=4(\mathrm{LH})$ & EN9 \\
\hline 3 & Spur Pinion $Z=35 \mathrm{~m}=4$ & EN24 \\
\hline 4 & Spur Gear $Z=75 \mathrm{~m}=4$ & EN9 \\
\hline 5 & $2^{\text {nd }}$ Spur Pinion $Z=23 \mathrm{~m}=6$ & EN24 \\
\hline 6 & Spur Gear $Z=67 \mathrm{~m}=6$ & EN9 \\
\hline 7 & Sun Pinion (Spur) $Z=18 \mathrm{~m}=6$ & 17CrNiMo6 \\
\hline 8 & Planet (Spur) $Z=26 \mathrm{~m}=6$ & 17CrNiMo6 \\
\hline 9 & Annulus Internal $Z=72 \mathrm{~m}=6$, External $\mathrm{Z}=76 \mathrm{~m}=9$ & EN9 \\
\hline 10 & Opening Closing Drum Shaft & EN19 \\
\hline 11 & Holding Drum Shaft & EN19 \\
\hline 12 & Spur Gear $Z=104 \mathrm{~m}=9$ & EN9 \\
\hline 13 & Spur Pinion $Z=24 \mathrm{~m}=9$ & EN24 \\
\hline 14 & Helical Gear Z = 79 m = 7 (RH) & EN9 \\
\hline 15 & Helical Pinion $\mathrm{Z}=20 \mathrm{~m}=7$ (LH) & EN24 \\
\hline 16 & Helical Gear Z = $80 \mathrm{~m}=5(\mathrm{LH})$ & EN9 \\
\hline 17 & Input Helical Pinion $\mathrm{Z}=19 \mathrm{~m}=5(\mathrm{RH})$ & EN24 \\
\hline
\end{tabular}

closing drum $\mathrm{a}_{1}$ rotates to lifting, i.e., counterclockwise while the hoisting drum $\mathrm{a}_{2}$ is immobile.

The closing rope $s_{1}$ is tightened, the movable crossmember goes upwards $\&$ the scoops cut into material as they are gradually brought together until their edges are tightly compressed.

In raising (Figure 6(c)), both drums rotate clockwise.

To dump the grab bucket(Figure 6(d)), the hoisting drum is braked \& closing drum revolves for descent (clockwise); this causes the bucket scoops to open under the action of their own weight $\&$ that of the material \& the contents are dumped.

This cycle continues through the duty hours.

\subsection{Normal Rating}

The normal ratings of the gears is the allowable continuous load for 12 hours running time per day.

\subsection{Duty Factor: (as per IS 4137)}

1) For Class III Crane: Duty factor for wear $=0.6$ Duty factor for strength $=1.4$

2) For Class IV Crane: Duty factor for wear $=0.7$ Duty factor for strength $=1.6$

\section{Checking Sun Gear Wear Stresses \& Bending Stresses Using IS 4460 Equations}

Capacity $=10$ tones. Speed $=20 \mathrm{~m} / \mathrm{min}$. From IS standards,

$$
\mathrm{KW} \text { ratings }=\frac{\text { Capacity } \times \text { Speed }}{6.12 \times \text { efficiency }}
$$

But Efficiency $=(0.95)^{n} \times(0.99)^{m}$ where $n=$ number of stages in gearbox $=9 . m=$ number of rotating sheaves between the rope drum $\&$ equalizer $=5$

Efficiency $=(0.95)^{9} \times(0.99)^{5}=0.5994$

$$
\mathrm{KW} \text { rating }=\frac{10 \times 20}{6.12 \times 0.5994}=54.524
$$

Considering $60 \%$ of ratings, $\mathrm{KW}$ rating $=0.6 \times 54.524$ $=32.71$

Further HP required $=32.71 / 0.735=44.47$ Assuming $\mathrm{HP}$ rating $=45$

\subsection{Calculations for Planetary System Speeds}

\author{
Refer: Figure 7 \\ Let, \\ $\mathrm{T}_{\mathrm{S}}=$ number of teeth on sun \\ $\mathrm{T}_{\mathrm{P}}=$ number of teeth on planet \\ $\mathrm{T}_{\mathrm{A}}=$ internal number of teeth on annulus
}


$\mathrm{N}_{\mathrm{A}}=$ speed of annulus

$\mathrm{N}_{\mathrm{S}}=$ speed of sun

$\mathrm{N}_{\mathrm{P}}=$ speed of planet

$\mathrm{N}_{\mathrm{C}}=$ speed of carrier

Different possible conditions for planetary gear drive system are mentioned in Table $\mathbf{3}$ and speed calculations for planetary unit are mentioned in Table 4

Calculations for opening closing mechanism is mentioned in Table 5

For mentioned gearbox, Speed calculations for planetary unit are mentioned in Table 6

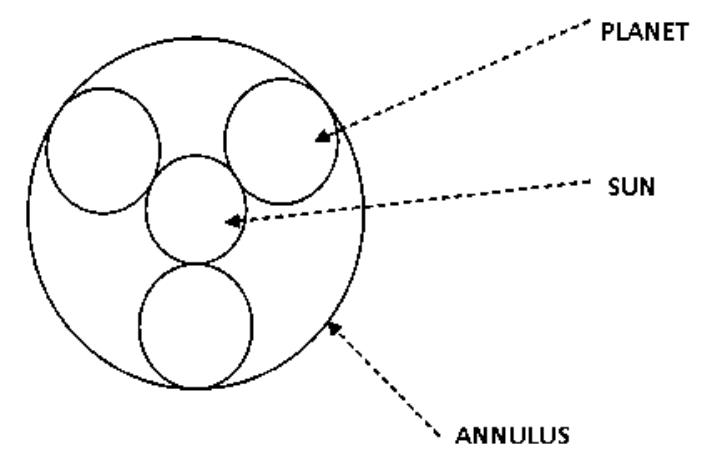

Figure 7. Schematic diagram of planetary gear train.
From the above table,

Speed of annulus $N_{A}$ is given as

$$
\begin{aligned}
& N_{A}=N_{C}\left(\frac{T_{A}+T_{S}}{T_{A}}\right)-\frac{N_{S} T_{S}}{T_{A}} \\
& N_{C}=\frac{N_{A} T_{A}+N_{S} T_{S}}{T_{A}+T_{S}}
\end{aligned}
$$

Table 3. Gear drive system design.

\begin{tabular}{ccccccc}
\hline I/P & Sun & Carrier & Ring & $\begin{array}{c}\text { Sun, } \\
\text { ring }\end{array}$ & $\begin{array}{c}\text { Carrier, } \\
\text { sun }\end{array}$ & $\begin{array}{c}\text { Ring } \\
\text { carrier }\end{array}$ \\
\hline $\mathrm{O} / \mathrm{P}$ & $\begin{array}{c}\text { Carrier } \\
\text { ring }\end{array}$ & $\begin{array}{c}\text { Sun, } \\
\text { Ring }\end{array}$ & $\begin{array}{c}\text { Carrier, } \\
\text { sun }\end{array}$ & Carrier & Ring & Sun \\
\hline
\end{tabular}

Table 4. Speed calculation for planetary unit.

\begin{tabular}{cccc}
\hline Sun & Planets & Carrier & Annulus \\
\hline+1 & +1 & +1 & +1 \\
-1 & $\frac{T_{S}}{T_{P}}$ & 0 & $\frac{T_{S}}{T_{P}} \times \frac{T_{P}}{T_{A}}=\frac{T_{S}}{T_{A}}$ \\
0 & $1+\frac{T_{S}}{T_{P}}$ & 1 & $1+\frac{T S}{T_{A}}$ \\
\hline
\end{tabular}

Table 5. Calculations for opening closing mechanism.

\section{H.P Ratings of Gears}

Eq. Running Time: $12.00 \mathrm{Hrs} /$ Day

Duty Class: 4

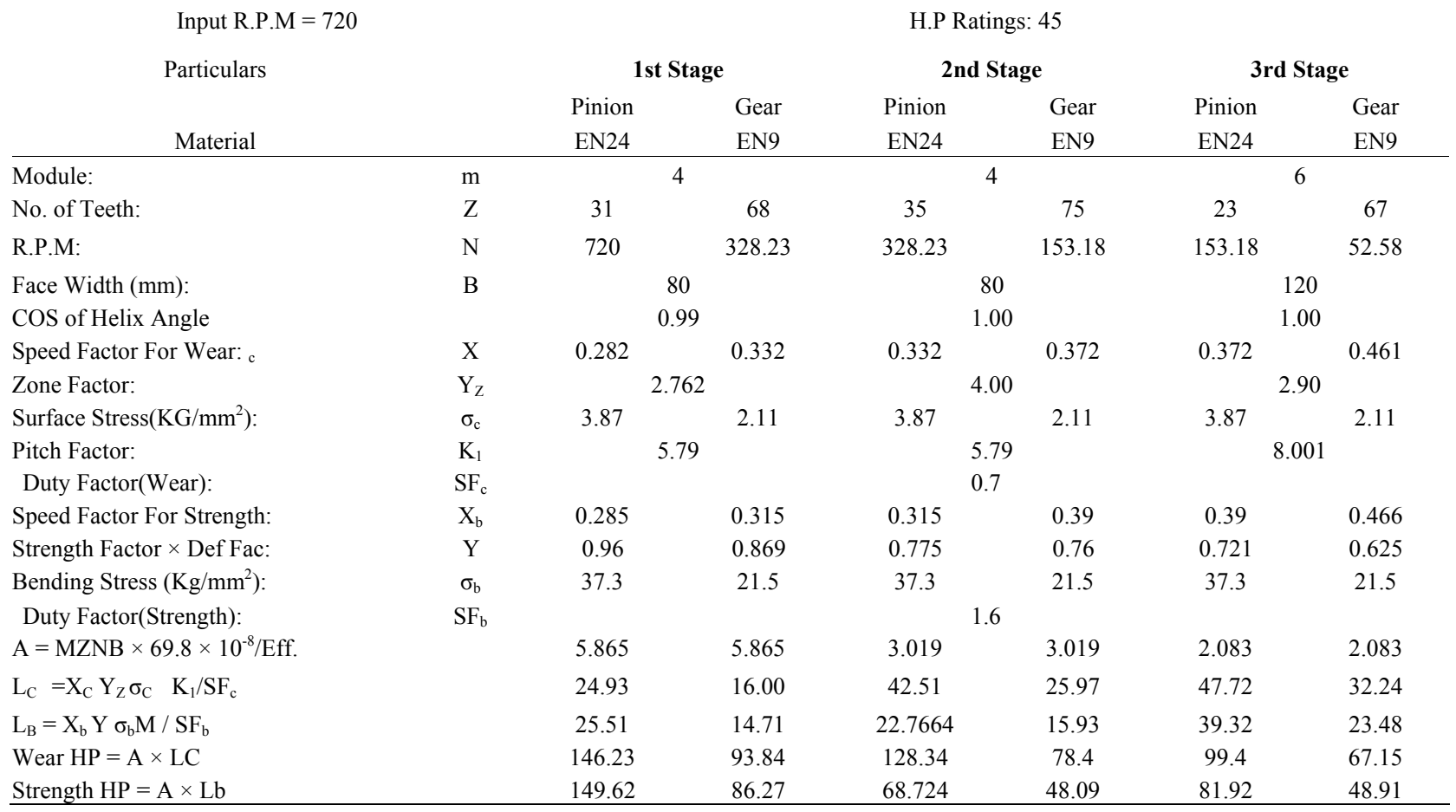


Table 6. Speed calculation for planetary unit.

\begin{tabular}{|c|c|c|c|c|}
\hline & Sun & Planets & Carrier & Annulus \\
\hline Carrier Fixed, Sun Rotated By $-\mathrm{N}_{\mathrm{s}}$ & $\mathrm{N}_{\mathrm{S}}$ & $-N_{S} \frac{T_{S}}{T_{P}}$ & 0 & $-N_{S} \frac{T_{S}}{T_{A}}$ \\
\hline Sun Fixed, Carrier Rotated By $-\mathrm{N}_{\mathrm{c}}$ & 0 & $N_{C} \times\left(1+\frac{T_{S}}{T_{P}}\right)$ & $\mathrm{N}_{\mathrm{C}}$ & $N_{C} \times\left(1+\frac{T_{S}}{T_{A}}\right)$ \\
\hline Total Motion & $\mathrm{N}_{\mathrm{S}}$ & $N_{C}\left(\frac{T_{P}+T_{S}}{T_{P}}\right)-\frac{N_{S} T_{S}}{T_{P}}$ & $\mathrm{~N}_{\mathrm{C}}$ & $N_{C}\left(\frac{T_{A}+T_{S}}{T_{A}}\right)-\frac{N_{S} T_{S}}{T_{A}}$ \\
\hline
\end{tabular}

When annulus is fixed, $N_{A}=0$

$$
N_{C}=\frac{N_{S} T_{S}}{T_{A}+T_{s}}=\frac{52.79 \times 18}{72+18}=10.52 \mathrm{rpm}
$$

Planet speed is given as,

$$
\begin{aligned}
& N_{\mathrm{P}}=N_{C}\left(\frac{T_{P}+T_{S}}{T_{P}}\right)-\frac{N_{S} T_{S}}{T_{P}} \\
& N_{\mathrm{P}}=10.52\left(\frac{26+18}{26}\right)-\frac{52.58 \times 18}{26} \\
& N_{P}=18.68 \mathrm{rpm}
\end{aligned}
$$

Calculations of HP for planetary system is mentioned in Table7

From the above table, speed of annulus $\mathrm{N}_{\mathrm{A}}$ is given as

$$
\begin{aligned}
& N_{A}=N_{C}\left(\frac{T_{A}+T_{S}}{T_{A}}\right)-\frac{N_{S} T_{S}}{T_{A}} \\
& N_{C}=\frac{N_{A} T_{A}+N_{S} T_{S}}{T_{A}+T_{S}}
\end{aligned}
$$

when annulus is fixed, $\mathrm{N}_{\mathrm{A}}=0$

$$
N_{C}=\frac{N_{S} T_{S}}{T_{A}+T_{s}}=\frac{52.79 \times 18}{72+18}=10.52 \mathrm{rpm}
$$

Planet speed is given as,

$$
\begin{aligned}
& N_{P}=N_{C}\left(\frac{T_{P}+T_{S}}{T_{P}}\right)-\frac{N_{S} T_{S}}{T_{P}} \\
& N_{P}=10.52\left(\frac{26+18}{26}\right)-\frac{52.58 \times 18}{26} \\
& N_{P}=18.68 \mathrm{rpm}
\end{aligned}
$$

But for planetary system

Strength of sun $=3 \times \mathrm{HP}_{\text {normal }}=3 \times 14.32=42.06 \mathrm{HP}$

Strength of sun in wear $=\mathrm{HP}_{\text {normal }} / 3=10.52 / 3=3.5 \mathrm{HP}$
Table 7. Calculation of HP for planetary system.

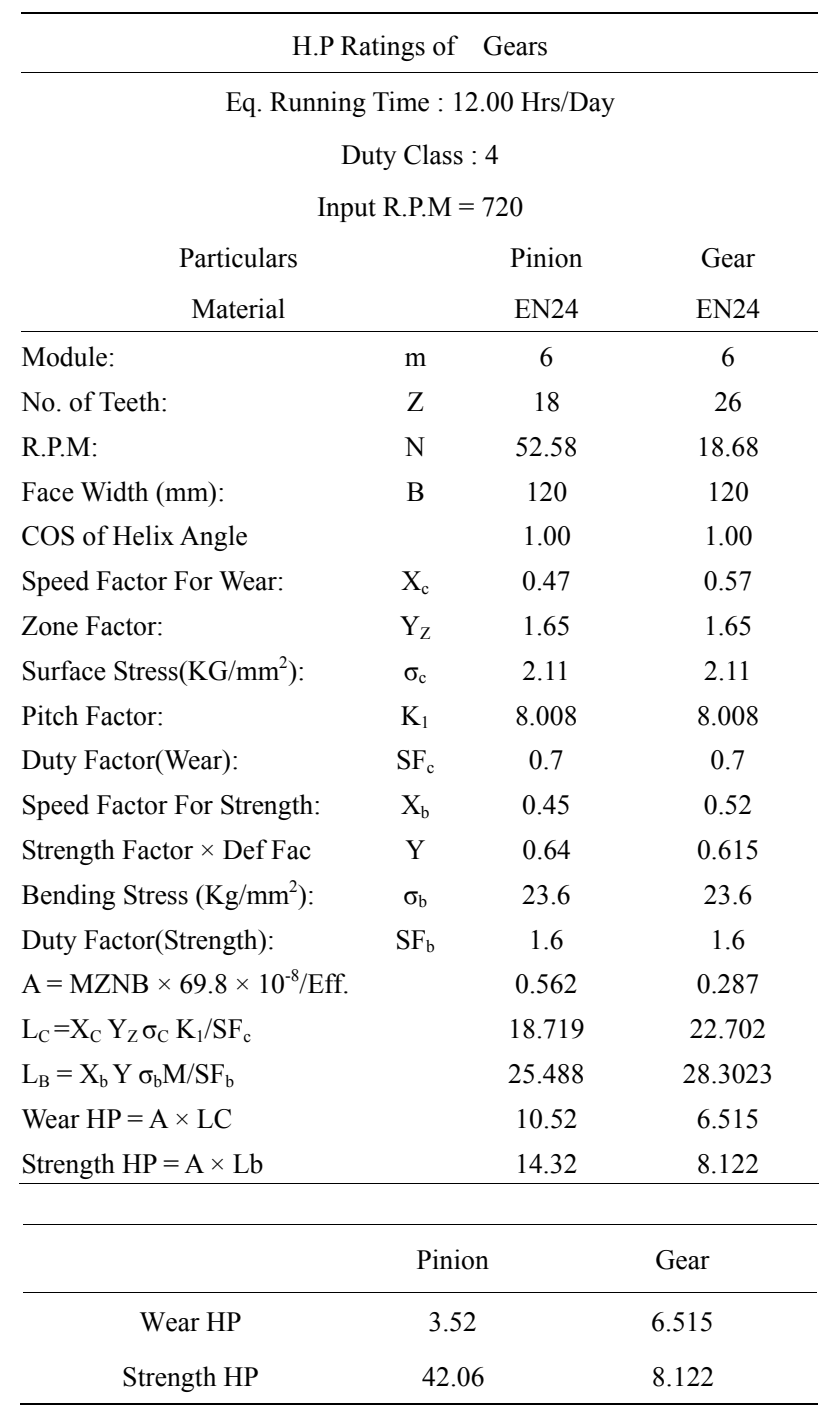

Hence HP rating for planetary system will be minimum of the above 4 HP values

$(\mathrm{HP})_{\text {Planetary }}=3.52$ 


\subsection{Digging Force Calculations}

Normal reaction

= Capacity

$=$ Volume of bucket $\times$ density of material

$=3.5 \times 1.1 \times 1000$

$=3850 \mathrm{Kg}$

$=3.85 \mathrm{~T}$

Assuming coefficient of frictions, $\mu=0.1$

Friction force $=\mu \mathrm{N}=0.1 \times 3.85=0.385 \mathrm{~T}$

Torque $=$ Force $\times$ Perpendicular distance

$$
\begin{aligned}
& =0.385 \times 1.8 \\
& =0.693 \mathrm{Tm}=693 \mathrm{~kg}-\mathrm{m}
\end{aligned}
$$

Time required to grab 1 Ton of slag

$=$ Displacement $/$ Velocity

$=1.8 / 20=0.09 \mathrm{~min}^{\circ}$

Speed $=0.5 / 0.09=5.56 \mathrm{rpm}$

From standard formulae,

$$
\begin{aligned}
& \mathrm{T}=\frac{736 \times \mathrm{HP}}{\mathrm{rpm}} \\
& 693=\frac{736 \times \mathrm{HP}}{5.56} \\
& \mathrm{HP}=\mathrm{HP}_{\text {induced }}=5.23
\end{aligned}
$$

Since, $\mathrm{HP}_{\text {induced }}>\mathrm{HP}_{\text {Planetary }}$

Design unsafe

Modified calculations for HP ratings for planetary gear system is mentioned in Table $\mathbf{8}$.

But for planetary system

Strength of sun $=3 \times \mathrm{HP}_{\text {normal }}$

$$
\begin{aligned}
& =3 \times 17.06 \\
& =51.18 \mathrm{HP}
\end{aligned}
$$

Strength of sun in wear $=\mathrm{HP}_{\text {normal }} / 3$

$$
\begin{aligned}
& =35.748 / 3 \\
& =11.916 \mathrm{HP}
\end{aligned}
$$

\begin{tabular}{ccc}
\hline & Pinion & Gear \\
Wear HP & 11.916 & 24.3 \\
Strength HP & 51.18 & 11.37 \\
\hline
\end{tabular}

Hence HP rating for planetary system will be minimum of the above $4 \mathrm{HP}$ values

$(\mathrm{HP})_{\text {Planetary }}=11.37$

\subsection{Digging Force Calculations}

Normal reaction

= Capacity

\begin{tabular}{|c|c|c|c|}
\hline \multicolumn{4}{|c|}{ H.P Ratings of Gears } \\
\hline \multicolumn{4}{|c|}{ Eq. Running Time : $12.00 \mathrm{Hrs/Day}$} \\
\hline \multicolumn{4}{|c|}{ Duty Class : 4} \\
\hline \multicolumn{4}{|c|}{ Input R.P.M = 720} \\
\hline \multicolumn{2}{|l|}{ Particulars } & \multicolumn{2}{|c|}{ 1st Stage } \\
\hline & & Pinion & Gear \\
\hline Material & & $\begin{array}{c}3 \% \text { Nickel } \\
\text { Steel }\end{array}$ & $\begin{array}{c}5 \% \text { Nickel } \\
\text { Steel }\end{array}$ \\
\hline Module & $\mathrm{m}$ & 6 & 6 \\
\hline No. of Teeth: & Z & 18 & 26 \\
\hline R.P.M: & $\mathrm{N}$ & 52.58 & 18.68 \\
\hline Face Width (mm): & B & 120 & 120 \\
\hline COS of Helix Angle & & 1.00 & 1.00 \\
\hline Speed Factor For Wear: & $\mathrm{X}_{\mathrm{c}}$ & 0.47 & 0.57 \\
\hline Zone Factor: & $\mathrm{Y}_{\mathrm{Z}}$ & 1.65 & 1.65 \\
\hline Surface Stress $\left(\mathrm{KG} / \mathrm{mm}^{2}\right)$ : & $\sigma_{\mathrm{c}}$ & 7.17 & 7.87 \\
\hline Pitch Factor: & $\mathrm{K}_{1}$ & 8.008 & 8.008 \\
\hline Duty Factor(Wear): & $\mathrm{SF}_{\mathrm{c}}$ & 0.7 & 0.7 \\
\hline Speed Factor For Strength: & $\mathrm{X}_{\mathrm{b}}$ & 0.45 & 0.52 \\
\hline Strength Factor $\times$ Def Fac & $\mathrm{Y}$ & 0.64 & 0.615 \\
\hline Bending Stress $\left(\mathrm{Kg} / \mathrm{mm}^{2}\right)$ : & $\sigma_{\mathrm{b}}$ & 28.12 & 33.07 \\
\hline Duty Factor(Strength): & $\mathrm{SF}_{\mathrm{b}}$ & 1.6 & 1.6 \\
\hline $\mathrm{A}=\mathrm{MZNB} \times 69.8 \times 10^{-8} / \mathrm{Eff}$ & & 0.562 & 0.287 \\
\hline $\mathrm{L}_{\mathrm{C}}=\mathrm{X}_{\mathrm{C}} \mathrm{Y}_{\mathrm{Z}} \sigma_{\mathrm{C}} \mathrm{K}_{1} / \mathrm{SF}_{\mathrm{c}}$ & & 63.61 & 84.67 \\
\hline $\mathrm{L}_{\mathrm{B}}=\mathrm{X}_{\mathrm{b}} \mathrm{Y} \sigma_{\mathrm{b}} \mathrm{M} / \mathrm{SF}_{\mathrm{b}}$ & & 30.36 & 39.65 \\
\hline Wear $\mathrm{HP}=\mathrm{A} \times \mathrm{LC}$ & & 35.748 & 24.3 \\
\hline Strength $\mathrm{HP}=\mathrm{A} \times \mathrm{Lb}$ & & 17.06 & 11.37 \\
\hline
\end{tabular}

$=$ Volume of bucket $\times$ density of material

$=3.5 \times 1.1 \times 1000$

$=3850 \mathrm{Kg}$

$=3.85 \mathrm{~T}$

Assuming coefficient of frictions, $\mu=0.1$
Table 8. Modified calculations. using pinion [3\% nickel steel with BHN 620 (case)] \& gear [5\% nickel steel with BHN 600(case)].

Friction force $=\mu \mathrm{N}=0.1 \times 3.85=0.385 \mathrm{~T}$

Torque $=$ Force $\times$ Perpendicular distance

$$
\begin{aligned}
& =0.385 \times 1.8 \\
& =0.693 \mathrm{Tm}=693 \mathrm{~kg}-\mathrm{m}
\end{aligned}
$$

Time required to grab 1 Ton of slag

$=$ Displacement $/$ Velocity

$=1.8 / 20=0.09 \mathrm{~min}$

Speed $=0.5 / 0.09=5.56 \mathrm{rpm}$

From standard formulae,

$$
\begin{aligned}
& T=\frac{736 \times H P}{\mathrm{rpm}} \\
& 693=\frac{736 \times H P}{5.56} \\
& \mathrm{HP}=\mathrm{HP}_{\text {induced }}=5.23 \\
& \text { Since, } \mathrm{HP}_{\text {induced }}<\mathrm{HP}_{\text {Planetary }}
\end{aligned}
$$

Design safe

Table 9 contains force calculations for mentioned grabbing crane hoist gear box. 
Table 9. Gear forces calculation.

\begin{tabular}{|c|c|c|c|c|c|c|c|c|c|c|c|c|c|c|c|}
\hline \multicolumn{2}{|c|}{ Particulars } & Module & $\begin{array}{l}\text { No of } \\
\text { Teeth }\end{array}$ & & Power (P & & $\mathrm{N}$ & Torque /Moment & $\begin{array}{c}\mathrm{PC} \\
\mathrm{D}\end{array}$ & $\mathrm{R}$ & $\mathrm{Ft}$ & $\cos \alpha$ & $\operatorname{SIN} \alpha$ & Fn & $\mathrm{Fr}$ \\
\hline \multicolumn{2}{|c|}{ Unit } & $\mathrm{mm}$ & No. & HP & Factor & $\mathrm{KW}$ & RPM & $\mathrm{N}-\mathrm{mm}$ & $\mathrm{mm}$ & $\mathrm{mm}$ & $\mathrm{N}$ & $\operatorname{COS} 20$ & SIN 20 & $\mathrm{~N}$ & $\mathrm{~N}$ \\
\hline \multicolumn{8}{|c|}{ Formulae } & $\begin{array}{c}(\mathrm{P} \times 60 \times 1000 \times 1000) \\
\quad /(2 \times(22 / 7) \times \mathrm{N})\end{array}$ & & $\mathrm{PCD} / 2$ & $\mathrm{Ft}=\mathrm{T} / \mathrm{R}$ & & & $\mathrm{Ft} / \mathrm{COS} \alpha$ & $\begin{array}{l}\text { Fn } \times \\
\operatorname{SIN} \alpha\end{array}$ \\
\hline \multirow{2}{*}{ 1st Stage } & Pinion & & 31 & 45 & 0.746 & 33.57 & 720 & 445056.8182 & 124 & 62 & 7178.3358 & 0.93969 & 0.34202 & 7639.025 & 2612.701 \\
\hline & Gear & & 68 & 45 & 0.746 & 33.57 & 328.23 & 976269.412 & 272 & 136 & 7178.4516 & 0.93969 & 0.34202 & 7639.149 & 2612.743 \\
\hline \multirow{2}{*}{ 2nd Stage } & Pinion & \multirow[b]{2}{*}{4} & 35 & 45 & 0.746 & 33.57 & 328.23 & 976269.412 & 140 & 70 & 13946.706 & 0.93969 & 0.34202 & 14841.77 & 5076.186 \\
\hline & Gear & & 75 & 45 & 0.746 & 33.57 & 153.18 & 2091923.94 & 300 & 150 & 13946.16 & 0.93969 & 0.34202 & 14841.19 & 5075.987 \\
\hline \multirow{2}{*}{ 3rd Stage } & Pinion & \multirow{2}{*}{6} & 23 & 45 & 0.746 & 33.57 & 153.18 & 2091923.94 & 138 & 69 & 30317.738 & 0.93969 & 0.34202 & 32263.46 & 11034.75 \\
\hline & Gear & & 67 & 45 & 0.746 & 33.57 & 52.58 & 6094349.735 & 402 & 201 & 30320.148 & 0.93969 & 0.34202 & 32266.03 & 11035.63 \\
\hline \multirow{3}{*}{$\begin{array}{l}\text { 4th Stage } \\
\text { (Planetary) }\end{array}$} & Sun & 6 & 18 & 45 & 0.746 & 33.57 & 52.58 & 6094349.735 & 108 & 54 & 112858.33 & 0.93969 & 0.34202 & 120101.3 & 41077.07 \\
\hline & $\begin{array}{l}\text { Planetary } \\
\text { (3nos) }\end{array}$ & 6 & 26 & 45 & 0.746 & 33.57 & 18.68 & 17154224.26 & 156 & 78 & 219925.95 & 0.93969 & 0.34202 & 234040.3 & 80046.5 \\
\hline & $\begin{array}{l}\text { Planetary } \\
\text { (1 No) }\end{array}$ & 6 & 26 & & & & & & & & 73308.651 & & & 78013.44 & 26682.17 \\
\hline
\end{tabular}

\section{Theoretical Stress \& Deflection Calculation}

\subsection{Bending Stress}

$\sigma_{\mathrm{b}}=$ Bending Stress

The classic method of estimating the bending stresses in a gear tooth is the Lewis equation. It models a gear tooth taking the full load at its tip as a simple cantilever beam. Refer: Figure 8

Lewis Bending Stress From,

$$
\sigma_{\mathrm{b}}=\frac{W t P \mathrm{~d}}{F Y}=\frac{W t \pi}{m F Y}
$$

where:

$\mathrm{Wt}$ is the tangential load

$\mathrm{Pd}$ is the diametral pitch

$\mathrm{F}$ is the face width

$\mathrm{Y}$ is the Lewis form factor

$\mathrm{m}$ is the module

Since tangential load

$\mathrm{Ft}=\mathrm{Wt}=112858.33$ act on 3 no. teeth,

hence tangential load

$\mathrm{Ft}=\mathrm{Wt}$ act on single teeth $=112858.33 / 3=$ $37619.443 \mathrm{~N}$

$\mathrm{Wt}=37619.443 \mathrm{~N}$

$\mathrm{F}$ is the face width $=120 \mathrm{~mm}$

$\mathrm{Y}$ is the Lewis form factor for 18 No. Teeth $=0.308$

$\mathrm{m}$ is module $=6 \mathrm{~mm}$

$\sigma_{\mathrm{b}}$ Bending Stress,

$$
\begin{aligned}
& \sigma_{\mathrm{b}}=\frac{W t \pi}{m F Y} \\
& \sigma_{\mathrm{b}}=\frac{37619.443 \times \pi}{6 \times 120 \times 0.308}
\end{aligned}
$$

$$
\sigma_{\mathrm{b}}=532.94 \mathrm{~N} / \mathrm{mm}^{2}
$$

\subsection{Shear Stress}

$\sigma_{\mathrm{s}}=$ Shear Stress

$$
\begin{aligned}
& \sigma_{\mathrm{s}}=\frac{\text { Load }}{\text { Area }}=\frac{P}{A}=\frac{F t}{A} \\
& \sigma_{\mathrm{s}}=\frac{F t}{\mathrm{~b} \times \text { Tooth Width }} \\
& \sigma_{\mathrm{s}}=\frac{F t}{b \times(\pi \times \mathrm{m}) / 2}
\end{aligned}
$$

Since tangential load

$\mathrm{Ft}=\mathrm{Wt}=112858.33$ act on 3 no. teeth,

hence tangential load

$\mathrm{Ft}=\mathrm{Wt}$ act on single teeth

$$
=112858.33 / 3=37619.443 \mathrm{~N}
$$

$$
\sigma_{\mathrm{s}}=\frac{F t}{b \times(\pi \times \mathrm{m}) / 2}
$$

$$
\sigma_{\mathrm{s}}=\frac{37619.443}{120 \times(\pi \times 6) / 2}
$$

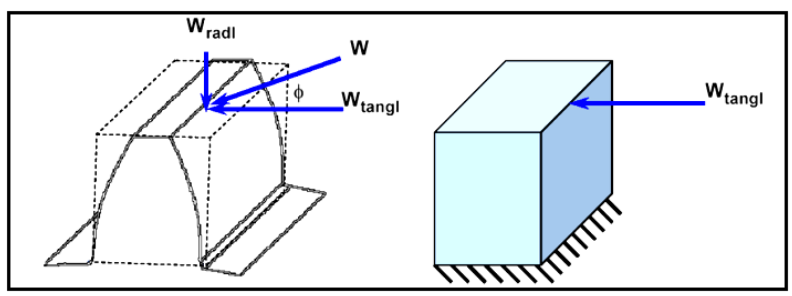

Figure 8. Tooth forces in spur gear. 
$\sigma_{\mathrm{s}}=33.2495077 \mathrm{~N} / \mathrm{mm}^{2}$

\subsection{Wear Stress}

$\sigma_{c}=$ Wear Stress

$$
\begin{aligned}
& \sigma_{\mathrm{c}}=0.74 \frac{i+1}{a} \sqrt{\frac{i+1}{i b}} \sqrt{E\left[M_{t}\right]} \\
& a=\text { Centre to centre distance }=132 \mathrm{~mm} \\
& i=\text { Gear Ratio }=2.81 \\
& E=\text { Modulus of Elasticity }=2 \times 10^{5} \mathrm{~N} / \mathrm{mm}^{2} \\
& \sigma_{\mathrm{c}}=0.74 \frac{2.81+1}{132} \sqrt{\frac{2.81+1}{2.81 \times 120}} \sqrt{2 \times 10^{5}\left[6094349.735_{t}\right]} \\
& \sigma_{\mathrm{c}}=2509.154561 \mathrm{~N} / \mathrm{mm}^{2}
\end{aligned}
$$

\subsection{Deflection}

$$
\begin{aligned}
& \text { Deflection }=\mathrm{A} / \mathrm{B} \\
& \begin{array}{l}
\text { Where }, \\
\mathrm{A}=\mathrm{Wt} \times \mathrm{L}^{3} \\
\mathrm{Wt}=\text { Tangential load } \\
\mathrm{Wt}=37619.443 \mathrm{~N} \\
\mathrm{~L}=\mathrm{H}=\text { Tooth Height } \\
=2.25 \times \text { Module } \\
=2.25 \times 6 \\
=13.5 \mathrm{~mm}
\end{array}
\end{aligned}
$$

Therefore,

$$
\begin{aligned}
& \mathrm{A}=37619.443 \times 13.5^{3} \\
& \mathrm{~A}=92557936.61 \\
& \&, \mathrm{~B}=3 \times \mathrm{E} \times \mathrm{I} \\
& \mathrm{E}=\text { Modulus of Elasticity } \\
& \mathrm{E}=2 \times 10^{5} \mathrm{~N} / \mathrm{mm}^{2} \\
& \mathrm{I}=\text { Moment of Inertia } \\
& \mathrm{I}=\left(\mathrm{a} \times \mathrm{b}^{3}\right) / 12 \\
& \mathrm{~A}=\text { Face Width } \\
& \mathrm{a}=120 \mathrm{~mm} \\
& \mathrm{~b}=\text { Tooth Width } \\
& \mathrm{b}=(\pi / 4) \times \text { Module } \\
& \mathrm{b}=(\pi / 4) \times 6 \\
& \mathrm{~b}=4.714 \mathrm{~mm} \\
& \text { Hence } \\
& \mathrm{I}=120 \times 4.714^{3} \\
& \mathrm{I}=4190.90379 \mathrm{~mm}^{4} \\
& \text { Therefore } \\
& \mathrm{B}=3 \times 2 \times 10^{5} \times 4190.90379 \\
& \mathrm{~B}=2514542274 \\
& \text { Therefore } \\
& \text { Deflection }=92557936.61 / 2514542274 \\
& \text { Deflection }=0.03680906 \mathrm{~mm}
\end{aligned}
$$

\section{Geometrical Modeling}

Consider the involute spur Gear, gear tooth of circular fillet illustrated in Figure 9 where point $\mathrm{O}$ is the center of the gear, axis Oy is the axis of symmetry of the tooth $\&$ point B is the point where the involute profile starts (form the form circle rs). A is the point of tangency of the circular fillet with the root circle rf. Point D lying on (e2) OA represents the center of the circular fillet. Line (e3) is tangent to the root circle at A \& intersects with line (e1) at C. The fillet is tangent to the line (e1) at point E. Since it is always $\mathrm{rs}>\mathrm{rf}$, the proposed circular fillet can be implemented without exceptions on all spur gears irrelevant of number of teeth or other manufacturing parameters. A comparison of the geometrical shape of a tooth of circular fillet with that of standard (trochoidal) fillet is presented in Figures $\mathbf{1 0}$ and $\mathbf{1 1}$.

\section{Element Analysis}

A finite element model with a single tooth is considered for analysis. Gear material strength is a major consideration for the operational loading \& environment. In modern practice, the heat treated alloy steels are used to overcome the wear resistance. ANSYS version 10.0 software is used for analysis. In this work, heat treated alloy is taken for analysis. The gear tooth is meshed in 3-dimensional (3-D) solid 16 nodes 92 elements with fine mesh. SOLID92 has a quadratic displacement behavior \& is well suited to model irregular meshes.

For sun gear used in grab crane's hoist Figure 12 and 13 indicates the PRO-E (3-D View) of Trochodial and Circular Fillet Tooth respectively. Also Figures 14 and 15 indicates the Meshing of Trochodial and\& Circular Fillet Tooth respectively in ANSYS.

The material properties chosen for analysis are as follow

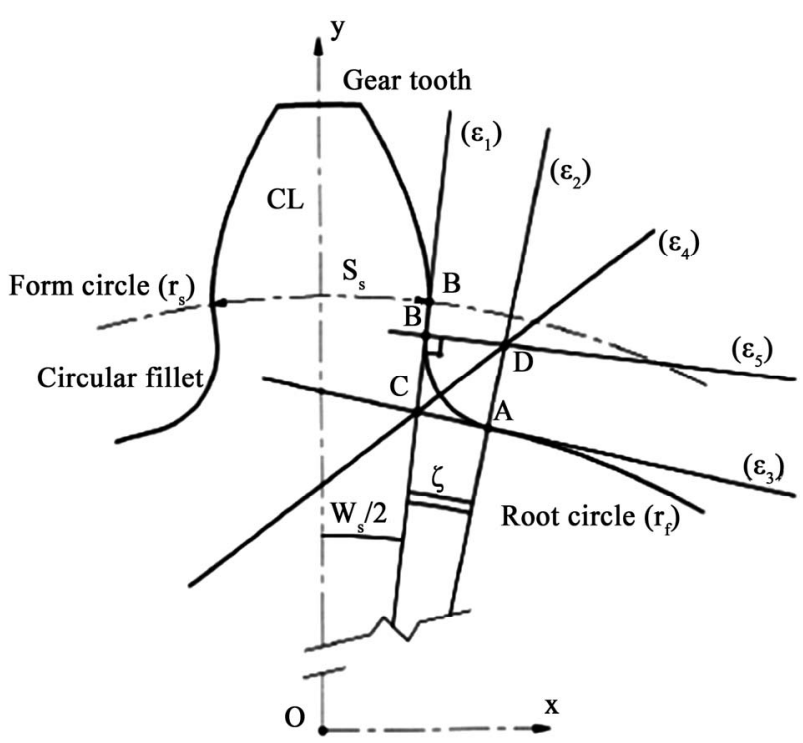

Figure 9. Geometry of the circular fillet. 


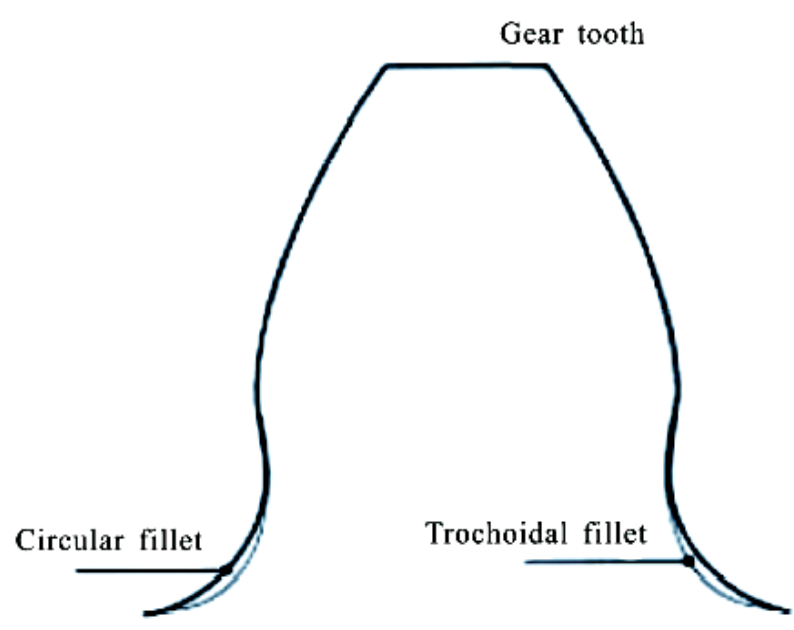

Figure 10. Superposition of circular fillet on a standard tooth.

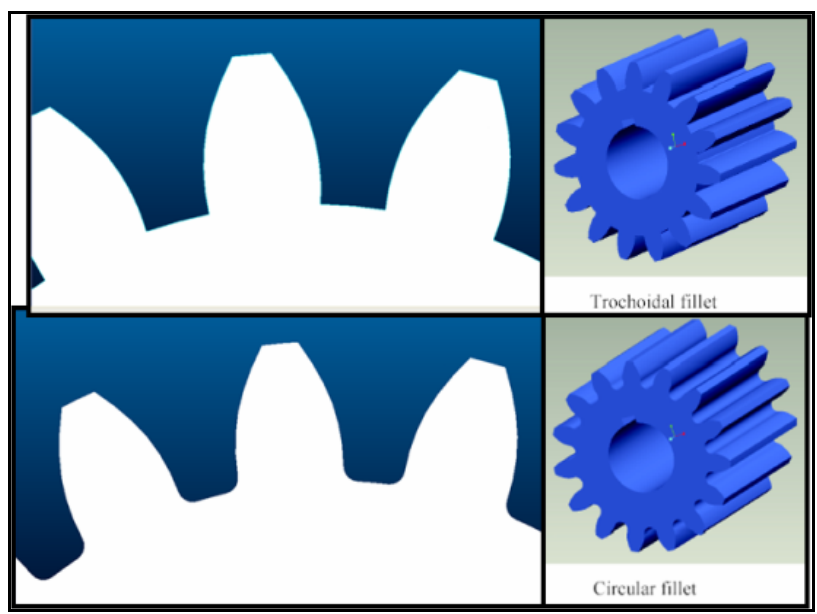

Figure 11. Geometrical modeling: trochoidal \& circular fillet spur gear.

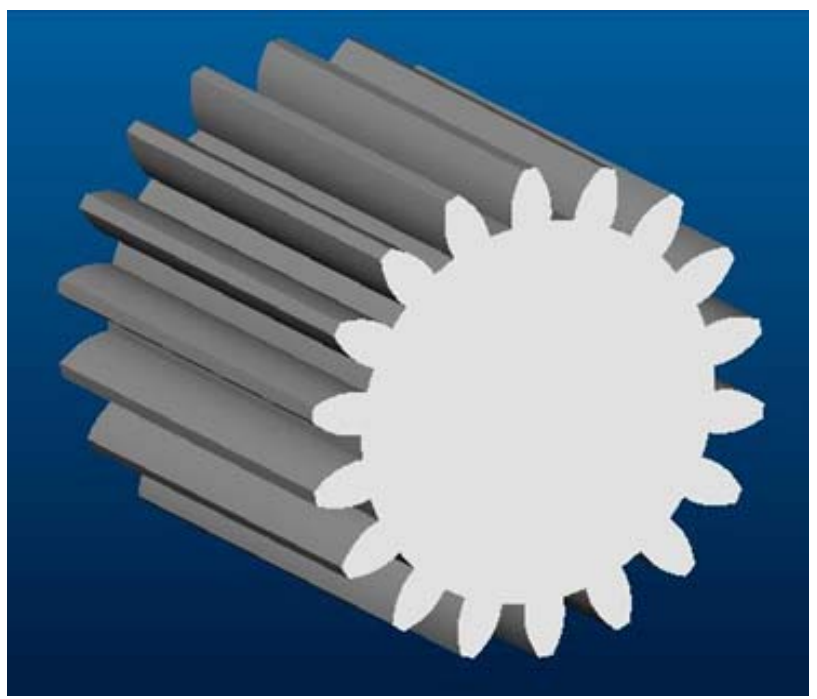

Figure 12. Trochodial fillet tooth sun gear in PRO-E (3D view).

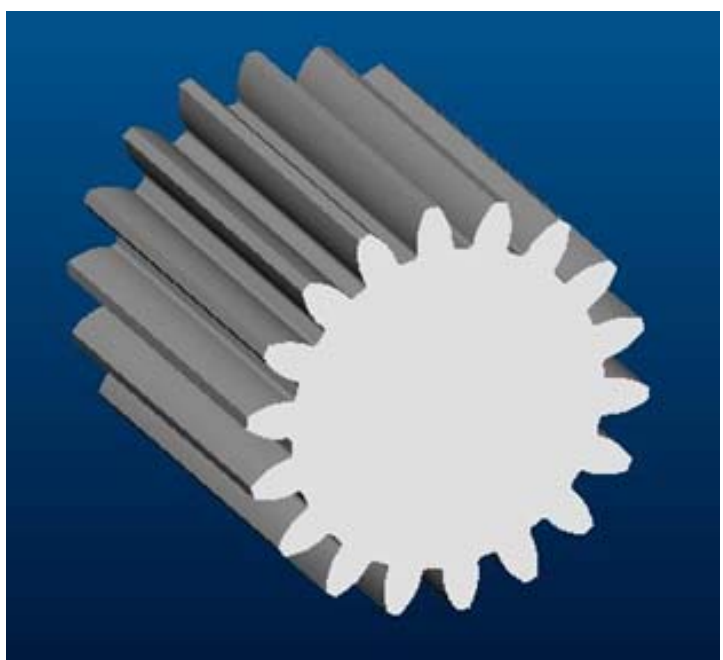

Figure 13. Circular fillet tooth sun gear in PRO-E (3D view).

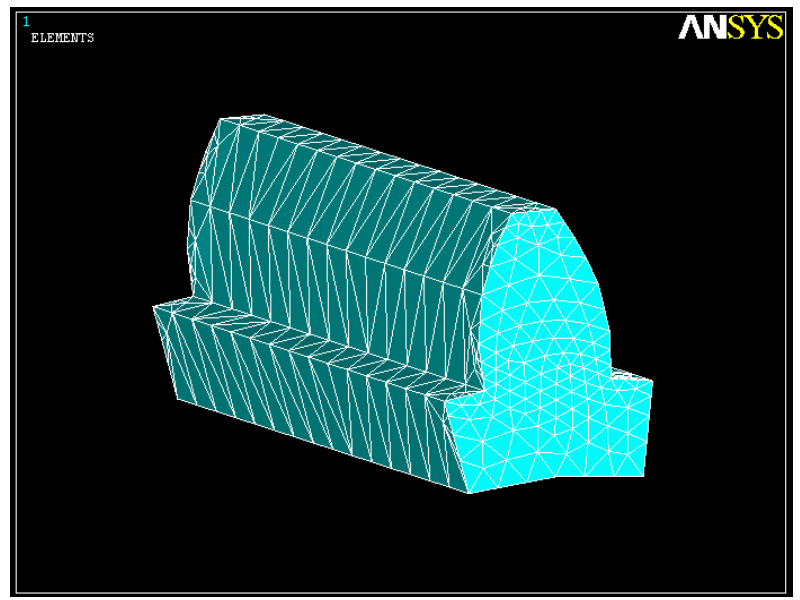

Figure 14. Meshing of trochodial tooth fillet sun gear in ANSYS (3D view).

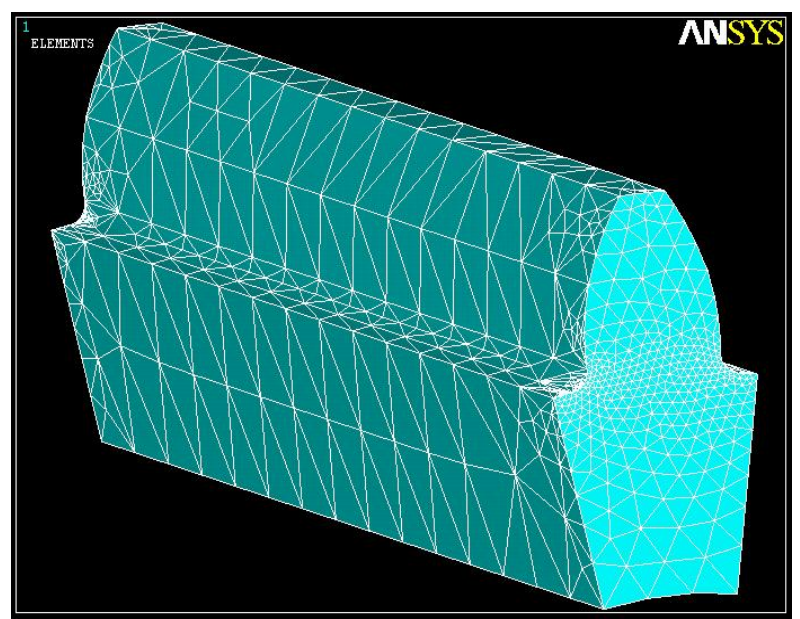

Figure 15. Meshing of circular fillet tooth sun gear in ANSYS (3D view). 


\section{Material properties.}

Gear material (Sun Gear): [3\% nickel steel with BHN 620 (case)]

Density: $7800 \mathrm{~kg} / \mathrm{m}^{3}$

Young's modulus: $2 \times 10^{5} \mathrm{~N} / \mathrm{mm}^{2}$

Poisons ratio: 0.3

Yield strength: $28.12 \mathrm{~kg} / \mathrm{mm}^{2}$

\section{Results}

Refer Figures 16-23 for ANSYS. Analysis for bending, shear, wear stress $\&$ deflection of sun gear used in grab crane's hoist. All results summarized in Table 10.

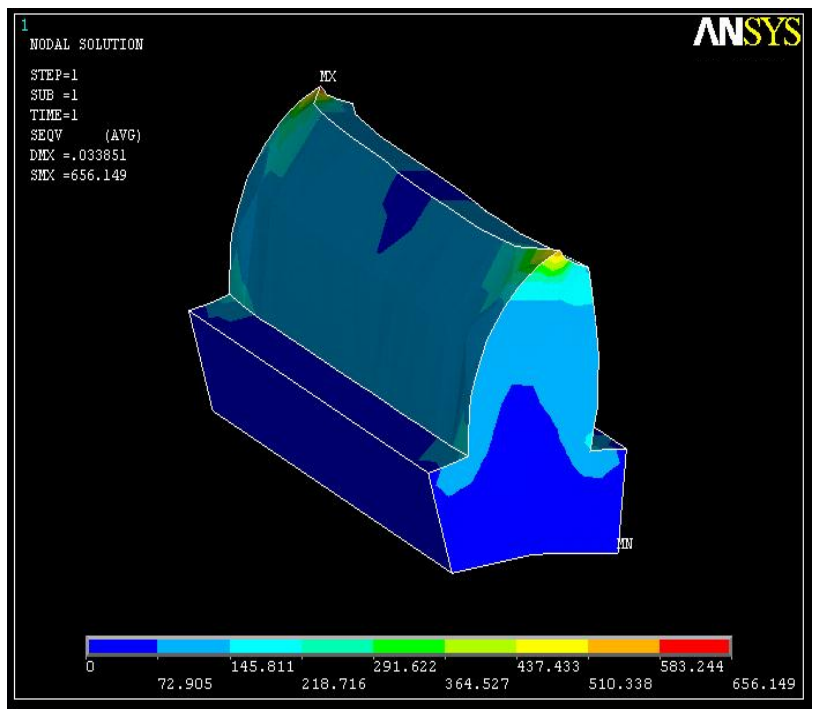

Figure 16. Bending stress trochoidal fillet result: maximum induced $\sigma_{\mathrm{b}}=656.149 \mathrm{~N} / \mathrm{mm}^{2}$.

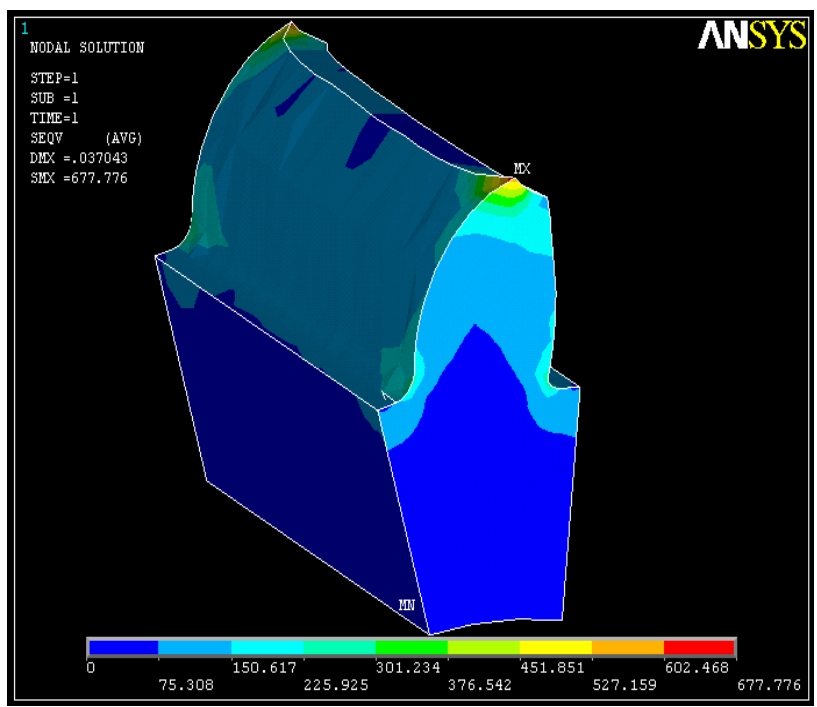

Figure 17. Bending stress circular fillet radius: maximum induced $\sigma_{b}=677.776 \mathrm{~N} / \mathrm{mm}^{2}$.

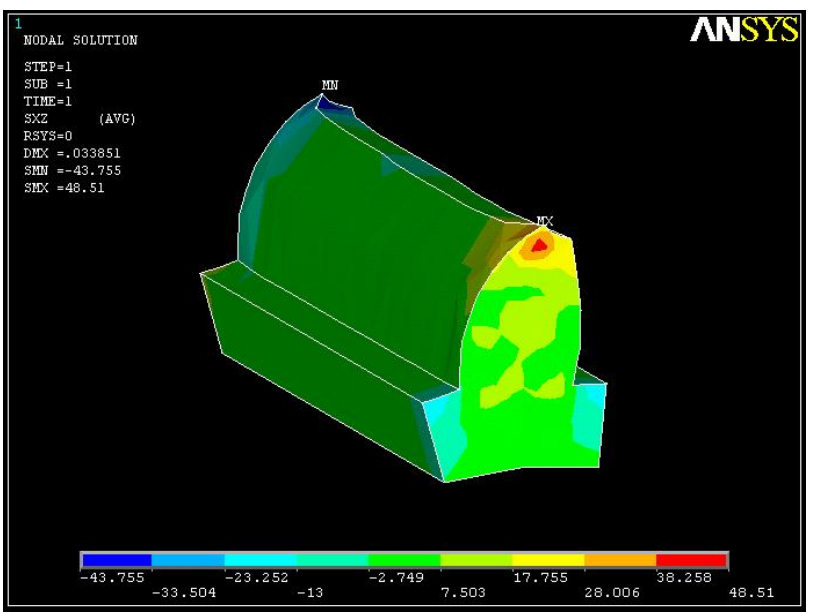

Figure 18. Shear stress-trochoidal fillet result: maximum induced $\sigma_{\mathrm{s}}=48.51 \mathrm{~N} / \mathrm{mm}^{2}$.

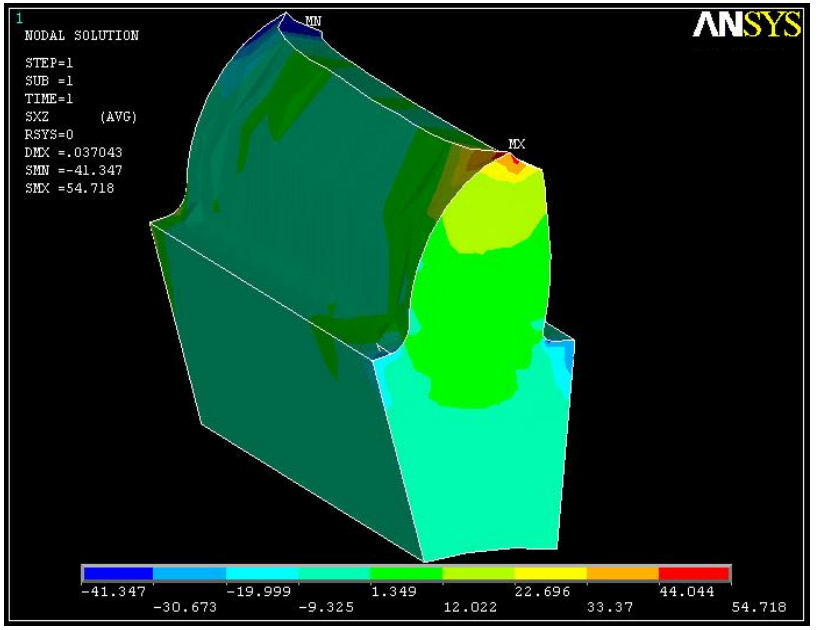

Figure 19. Sher stress circular fillet result: maximum induced $\sigma_{\mathrm{s}}=54.71 \mathrm{~N} / \mathrm{mm}^{2}$.

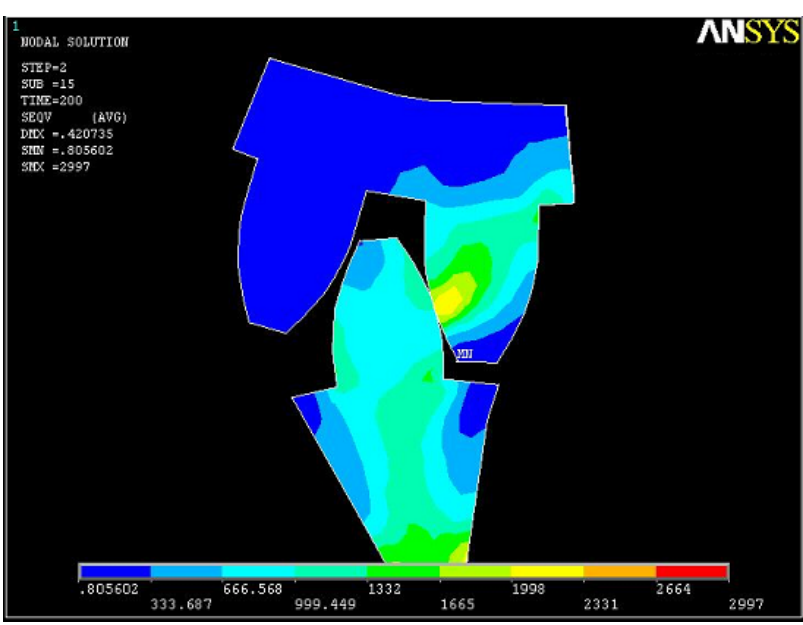

Figure 20. Wear stress trochoidal fillet result:- maximum wear $\sigma_{c}=1998 \mathrm{~N} / \mathrm{mm}^{2}$. 


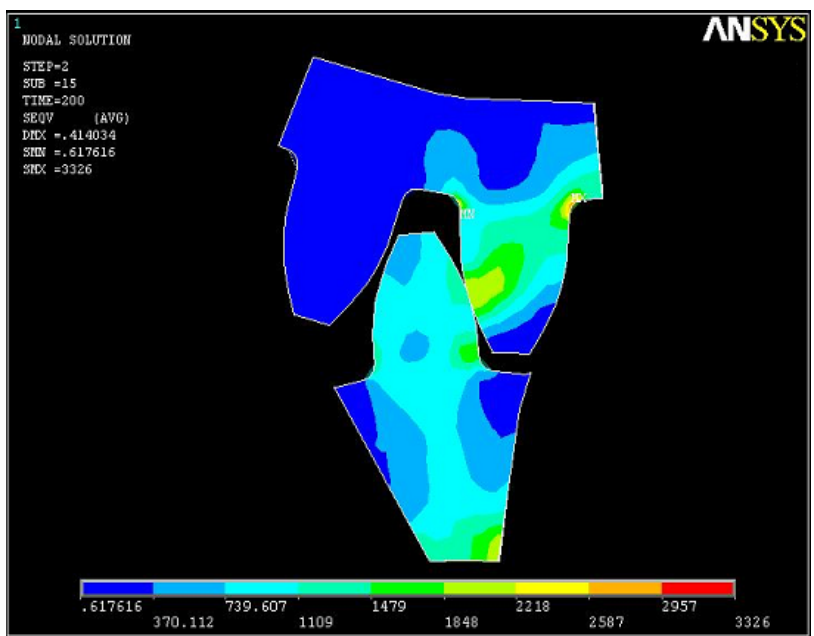

Figure 21. Wear stress circular fillet result: maximum wear $\sigma_{\mathrm{c}}=1848 \mathrm{~N} / \mathrm{mm}^{2}$.

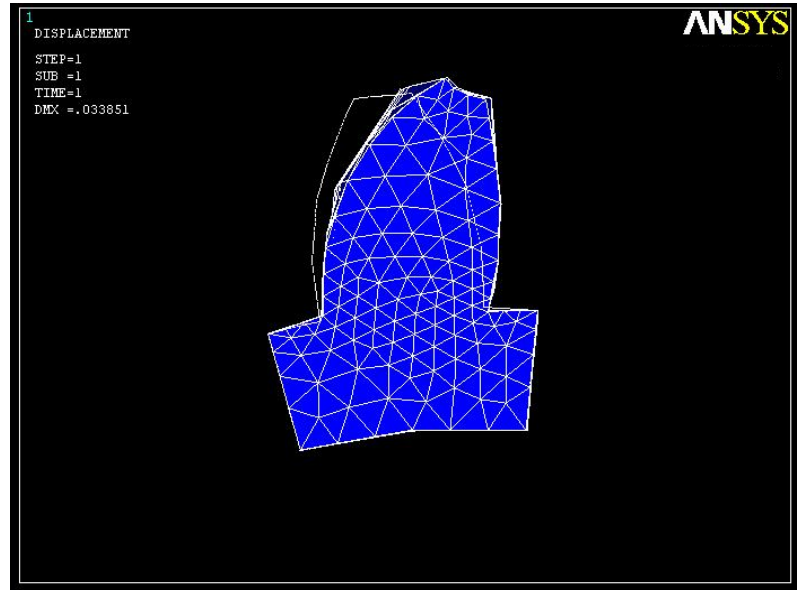

Figure 22. Deflection trochoidal fillet result: deflection $=$ 0.033851 mm.

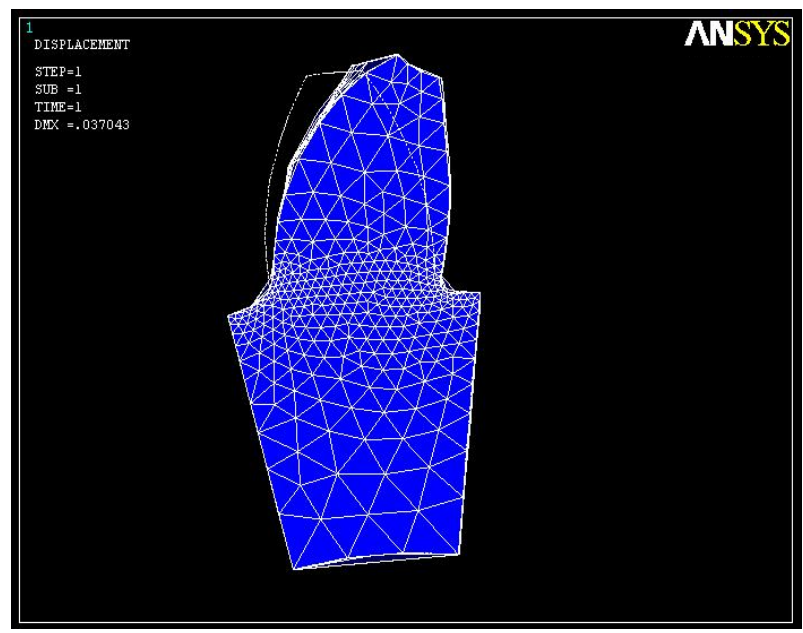

Figure 23. Deflection circular fillet result: deflection = $0.037043 \mathrm{~mm}$.
Table 10. Bending, shear \& wear stresses \& deflection result.

\begin{tabular}{cccc}
\hline Analysis & $\begin{array}{c}\text { Theoretical } \\
\text { Value }\end{array}$ & $\begin{array}{c}\text { For Trochoidal } \\
\text { Fillet Radius }\end{array}$ & $\begin{array}{c}\text { For Circular } \\
\text { Fillet Radius }\end{array}$ \\
\hline $\begin{array}{c}\text { Bending Stress } \\
\left(\mathrm{N} / \mathrm{mm}^{2}\right)\end{array}$ & 532.94 & 656.149 & 677.776 \\
$\begin{array}{c}\text { Shear Stress (N/ } \\
\left.\mathrm{mm}^{2}\right)\end{array}$ & 33.24 & 48.51 & 54.71 \\
$\begin{array}{c}\text { Wear Stress (N/ } \\
\left.\mathrm{mm}^{2}\right)\end{array}$ & 2509.1546 & 1998 & 1848 \\
$\begin{array}{c}\text { Deflection (mm) } \\
\text { Defle }\end{array}$ & 0.0368090 & 0.033851 & 0.037043 \\
\hline
\end{tabular}

\section{Conclusions}

ANSYS results for various stresses \& deflection are nearer to theoretical values for sun gear of planetary gear system of grabbing crane. There is appreciable reduction in bending \& shear stress value for trochoidal root fillet design in comparison to that of stresses values in circular root fillet design. Also there is increase in wear stress value for trochoidal root fillet design in comparison to that of stresses values in circular root fillet design. The investigation result infers that the deflection in trochoidal root fillet is also less comparing to the circular root fillet gear tooth. However, from the foregoing analysis it is also found that the circular fillet design is more optimum for lesser number of teeth in pinion \& trochoidal fillet design is more suitable for higher number of teeth in gear (more than 17 teeth) \& whatever may be the pinion speed. In addition to that the ANSYS results indicates that the gears with trochoidal root fillet design will result in better strength, reduced bending stress $\&$ also improve the fatigue life of gear material. Further work shall be done to calculate actual theoretical value of trochoidal \& circular root fillet gear tooth \& then carried out comparison with ANSYS results.

\section{References}

[1] S. Sankar, M. S. Raj and M. Nataraj, "Profile Modification for Increasing the Tooth Strength in Spur Gear Using CAD," Journal of Mechanical Engineering Science, Vol. 2, No. 9, 2010, pp. 740-749.

[2] Y. A. Tesfahunegn and F. Rosa, "The Effects of the Shape of Tooth Profile Modification on the Transmission Error Bending and Contact Stress of Spur Gears," Journal of Mechanical Engineering Science, Vol. 224, No. 8, 2010, pp. 1749-1758.

[3] C.-F. Tsai, T.-L. Liang and S.-C. Yang, "Mathematical Model of the Planetary Gear Mechanism with Double Circular-Arc Teeth," Transactions of the CSME Ide la SCGM, Vol. 32, No. 2, 2008, pp. 267-282 
[4] R. Patchigolla and Y. P. Singh, "Finite Element Analysis of Large Spur Gear Tooth and Rim with and without Web Effects-Part I and II," The 2006 ASEE Gulf-Southwest Annual Conference, Southern University and A \& M College.
[5] F. L. Litvin, A. Fuentes, D. Vecchiato and I. GonzalezPerez, "New Design and Improvement of Planetary Gear Trains," NASA/CR, 2004, pp. 1-30. 